\title{
Forced Expiratory Volume in 0.1 Second to FVC Ratio Measurement
}

National Cancer Institute

\section{Source}

National Cancer Institute. Forced Expiratory Volume in 0.1 Second to FVC Ratio

Measurement. NCI Thesaurus. Code C139244.

The ratio of the volume of gas that is forcibly exhaled during the first 0.1 seconds following maximal inhalation to that of the forced vital capacity. 